\title{
Bacteria capture using artificial magnetic cilia
}

\author{
Srinivas Hanasoge ${ }^{1}$, Alexander Alexeev ${ }^{1}$, Ynes Ortega ${ }^{2}$, Marilyn Erikson², Peter J. Hesketh ${ }^{1}$ \\ ${ }^{1}$ Woodruff School of Mechanical Engineering, Georgia Tech, Atlanta, GA, USA, \\ ${ }^{2}$ College of Agricultural and Environmental Sciences, University of Georgia, Griffin, GA, USA \\ Corresponding author: peter.hesketh@me.gatech.edu
}

\begin{abstract}
:
Microorganisms use hair like cilia on their surface to perform many biophysical functions. Most important among them is the feeding mechanism, in which the organism creates fluid flow to trap particles and feed on them. Natural cilia are also used to sort particles before feeding. In this work, we demonstrate artificial cilia capable of capturing particles in a microfluidic device. Elastic NiFe thin filaments are used as cilia, which extend away from the substrate. The bio-mimetic oscillations of the cilia, enhances fluid mixing and therefore increases the probability of particle capture. This can be particularly useful for target pre-concentration and detection of species of interest. We demonstrate the capture of salmonella bacteria on the surface of the cilia, which is functionalized with antisalmonella. This target specific antibody enables specific capture of salmonella bacteria in a given sample. In general, a similar technique as demonstrated here can be used to capture any specific target.
\end{abstract}

Key words: Artificial cilia, Salmonella capture, bio-mimetic capture, magnetic cilia, BioMEMS.

\section{Introduction}

Isolating rare cells and pre-concentrating target species for analysis are important steps in detection and enumeration of bacteria and cancer cells. Typically, microfluidic devices use an antibody functionalized passive surface to capture target species dispersed in a fluid stream [1]. The efficiency of capture in such systems depend on the probability of target cells encountering the antibody-coated surface. The chance of such capture can be increased if the fluid is mixed by agitating the fluid flow near the coated surfaces. Furthermore, the capture efficiency can be increased if the active surface extends into the fluid and increase the surface area for capture.

In this work, we demonstrate anti-body immobilized artificial magnetic cilia for capturing bacteria in a microfluidic channel flow. The cilia oscillate to mix fluid and to create flow circulations that bring bacteria closer to the surface of cilia. Furthermore, antibody coated beating cilia sweep through a large area of fluid enhancing contact probability with target bacteria to facilitate their capture. Thus, the use of arrays of magnetic cilia can increase the capture efficiency of the target compared to passive surfaces.

\section{Methods}

In our experiments, we capture fluorescently tagged (GFP) Salmonella using anti-Salmonella coated cilia. Following the fabrication process detailed elsewhere [2]., we obtain magnetic cilia on a glass substrate as shown in Fig. $1 \mathrm{~b}, \mathrm{c}$. Such cilia, can be actuated using a simple rotating magnet (Fig. 1a). These cilia are capable of beating in a asymmetric, bio-mimetic fashion [2] and produce microfluidic pumping[3]. To create the capturing surface, we immobilize antibody coated microbeads on the surface of the cilia. To this end, the cilia are first immersed in $1 \%$ APTES solution. The silane group binds to the thin oxide layer on the $\mathrm{NiFe}$, and the amine group is protonated $-\mathrm{NH}_{3}^{+}$creating an overall positive charge on the cilia. Next, carboxylate microbeads coated with antisalmonella are introduced. The beads with the carboxyl group $-\mathrm{COO}^{-}$have a slight negative charge and, therefore bind to the surface of the cilia electrostatically. This procedure results in cilia covered with antibody-coated microbeads that are electrostatically bound to the surface[4]. Similarly, the bottom glass wall of the microchannel is also covered with antibodycoated microbeads. The anti-body coated glass is a passive surface and acts as a control. 
Comparing the number of cells captured on the cilia, and glass surface should give the effectiveness of the cilia. Fig. 2 shows the microbeads immobilized on the cilia surface and glass surface.

We introduce salmonella bacteria with a concentration of $10^{5} \mathrm{cells} / \mathrm{ml}$ in a microchannel with an array of cilia attached to the bottom channel wall. The cilia are actuated at $50 \mathrm{~Hz}$ for a period of 10 minutes during which $1 \mathrm{ml}$ of the bacteria sample flows through across the array.

\section{Results and Conclusions}

Fig. 3 shows images obtained using a fluorescent microscope that visualize the location of captured fluorescent bacteria on the cilia and channel substrate. The experiment reveals that the amount of GFP-Salmonella captured by the cilia is significantly larger than the amount of Salmonella captured on the bottom glass surface. Indeed, only a few cells can be seen sticking to the glass, whereas most of the cells are found on the cilia. Furthermore, the captured cells are localized on the edges of beating cilia where the highest local shear rates can be expected (Fig. 3b). Thus, the experiment demonstrates the increased capture efficiency of cilia compared to a passive surface. This shows the potential of using magnetic cilia to capture target species from a fluid for their effective isolation and preconcentration.

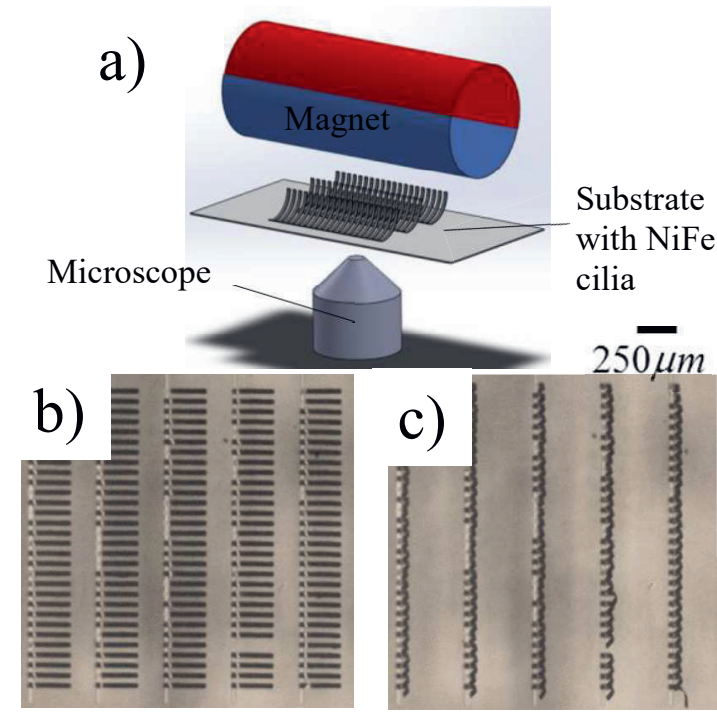

Fig. 1. a) Schematic of the magnetic actuation of the cilia. b), c) Microscope images of cilia actuated under a rotating magnetic field. Note the motion of cilia out of the plane of view. b) Shows the position of cilia parallel to the substrate. c) Shows the cilia standing upright, perpendicular to the substrate.

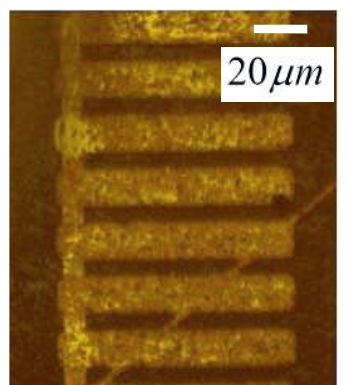

Fig. 2. Magnetic cilia with immobilized micro beads on the surface. The microbeads are coated with antisalmonella. This results in an antibody coated cilia surface.
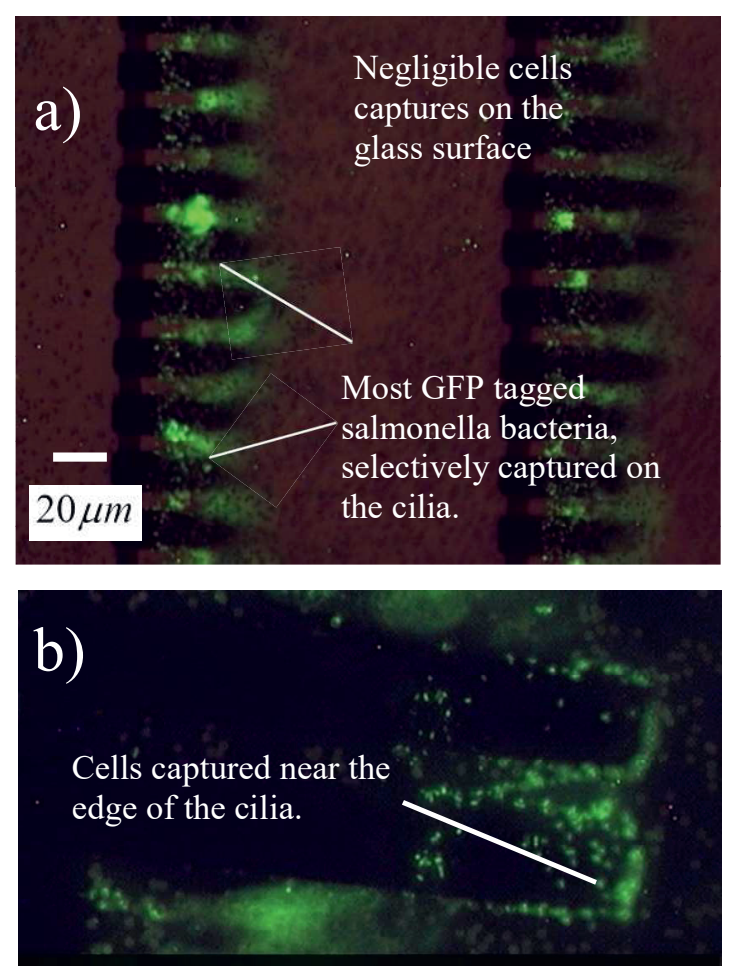

Fig. 3. Salmonella bacteria captured on the cilia surface. a) The concentration of captured cells is much larger on the cilia surface, as compared to the bottom glass surface. b) The cells are preferentially captured at the edges of the cilia.

\section{References}

[1] S. K. Murthy, A. Sin, R. G. Tompkins, and M. Toner, "Effect of flow and surface conditions on human lymphocyte isolation using microfluidic chambers," Langmuir ACS J. Surf. Colloids, vol. 20, no. 26, pp. 11649-11655, Dec. 2004.

[2] S. Hanasoge, M. Ballard, P. J. Hesketh, and A. Alexeev, "Asymmetric motion of magnetically actuated artificial cilia," Lab. Chip, vol. 17, no. 18, pp. 3138-3145, 2017.

[3] S. Hanasoge, P. J. Hesketh, and A. Alexeev, "Microfluidic pumping using artificial magnetic cilia," Microsyst. Nanoeng. in Press, 2018.

[4] V. Sivagnanam, A. Sayah, C. Vandevyver, and M. Gijs, "Micropatterning of proteinfunctionalized magnetic beads on glass using electrostatic self-assembly," Sens. Actuators B Chem., vol. 132, no. 2, pp. 361-367, Jun. 2008. 